\title{
An epidemiological survey of tumour or tumour like conditions in the scapula and periscapular region
}

\author{
Zeeshan Khan*, Adam M. Gerrish, and Robert J. Grimer \\ The Bone Tumour Unit, The Royal Orthopaedic Hospital, Bristol Road South, Northfield, Birmingham B31 2AP, UK
}

Received 23 May 2016, Accepted 16 August 2016, Published online 14 October 2016

\begin{abstract}
Introduction: The scapula is not an uncommon site for bone and soft tissue tumours and can be difficult to delineate on examination. Furthermore, these lesions can be potentially challenging to biopsy due to its close anatomical relationship with important structures. We present an epidemiological survey of all the scapular and periscapular lesions presenting to our institution.

Methodology: This was a retrospective study with data obtained from a prospectively held electronic database over a 30-year period. Demographic and clinical data was obtained and various subgroup analyses were performed.

Results: A total of 418 scapular lesions were included in the study where 132 lesions were found to be of soft tissue origin and 286 were osseous. Fifty-eight percent $(n=241)$ of all these lesions were malignant, of which $47 \%$ $(n=113)$ were primary sarcomas. The commonest malignant lesions were bone sarcomas $(n=96)$ followed by metastases $(n=88)$. The commonest primary bone sarcoma was chondrosarcoma $(45 \%)$, whereas the commonest soft tissue sarcoma was high grade undifferentiated pleomorphic sarcoma (18\%). The most common benign osseous and soft tissue lesions were osteochondroma (70\%) and lipoma (26\%), respectively. We noted that the incidence of malignancy increased with increasing age, however, the incidence of primary bone sarcomas was fairly consistent across different age groups.

Conclusion: Based on our findings we recommend that suspicious lesions arising from the scapula should be dealt with in a specialist sarcoma unit with involvement of a multidisciplinary team to offer appropriate management and advice for optimum outcome.
\end{abstract}

Key words: Scapula, Periscapular, Lesions, Tumours, Sarcoma.

\section{Introduction}

The shoulder girdle is comprised of the proximal humerus, scapula, clavicle and the surrounding soft tissues and is the third most common site for bone and soft tissue tumours $[1,2]$. In a report of 1853 cases of bone cancer, 3.6\% $(n=66)$ were found to originate from the scapula [3]. The scapula provides attachment to 17 muscles and is also affected by soft tissue sarcomas (STS) which can be difficult to delineate from bony pathology on examination. A further wide array of pathological conditions including metastatic deposits, haematological malignancies and non-malignant pathologies also present as suspicious scapula lesions.

Previous studies have shown that primary bone tumours of the scapula are more likely to be malignant than benign and the three most common subtypes of malignant bone tumours are osteosarcoma, chondrosarcoma and Ewing's

*Corresponding author: zeekl978@yahoo.co.uk sarcoma $[2,4,5]$. Although Ewing's sarcoma is the second most common malignant bone tumour of children and young adults, it occurs rarely in the scapula [4]. To our knowledge, very few cases of Ewing's sarcoma of scapula have been reported in the literature so far $[6,7]$.

The majority of the scapula forms by intramembranous ossification. Ossification of the body and the spine of the scapula occurs before birth, but ossification of the coracoid process, glenoid, acromion, vertebral border and inferior angle takes place throughout childhood, with the two ossification centres unifying at approximately 15 years of age.

The scapula presents itself as a challenging site to perform biopsies due to a number of factors. The most important are the neurovascular bundles and it is also important to avoid contamination of the non-involved muscular planes and injury to the chest wall [2]. Early diagnosis of scapula lesions is critical in improving outcomes, but this can be challenging due to the infrequency with which these conditions are encountered and the number of different pathologies affecting this area. 
Table 1. Distribution of different lesions of the scapula in various age groups.

\begin{tabular}{|c|c|c|c|c|c|c|c|c|c|c|}
\hline Diagnosis & $\begin{array}{c}0-9 \\
\text { years }\end{array}$ & $\begin{array}{l}10-19 \\
\text { years }\end{array}$ & $\begin{array}{l}20-29 \\
\text { years }\end{array}$ & $\begin{array}{c}30-39 \\
\text { years }\end{array}$ & $\begin{array}{c}40-49 \\
\text { years }\end{array}$ & $\begin{array}{l}50-59 \\
\text { years }\end{array}$ & $\begin{array}{c}60-69 \\
\text { years }\end{array}$ & $\begin{array}{l}70-79 \\
\text { years }\end{array}$ & $\begin{array}{c}80-89 \\
\text { years }\end{array}$ & Total \\
\hline Benign bone tumour & 23 & 22 & 25 & 17 & 6 & 3 & 3 & 2 & 1 & 102 \\
\hline Benign soft tissue tumour & 1 & 5 & 2 & 6 & 9 & 11 & 9 & 7 & 0 & 50 \\
\hline Bone sarcoma & 7 & 18 & 12 & 9 & 11 & 14 & 12 & 11 & 2 & 96 \\
\hline Haematological malignancy & 0 & 1 & 1 & 6 & 6 & 8 & 9 & 7 & 2 & 40 \\
\hline Metastasis & 0 & 0 & 0 & 4 & 12 & 11 & 27 & 23 & 11 & 88 \\
\hline Non-oncological diagnosis & 1 & 4 & 3 & 1 & 6 & 3 & 5 & 1 & 1 & 25 \\
\hline Soft tissue sarcomas & 1 & 0 & 3 & 1 & 4 & 1 & 4 & 2 & 1 & 17 \\
\hline Total & 33 & 50 & 46 & 44 & 54 & 51 & 69 & 53 & 18 & 418 \\
\hline
\end{tabular}

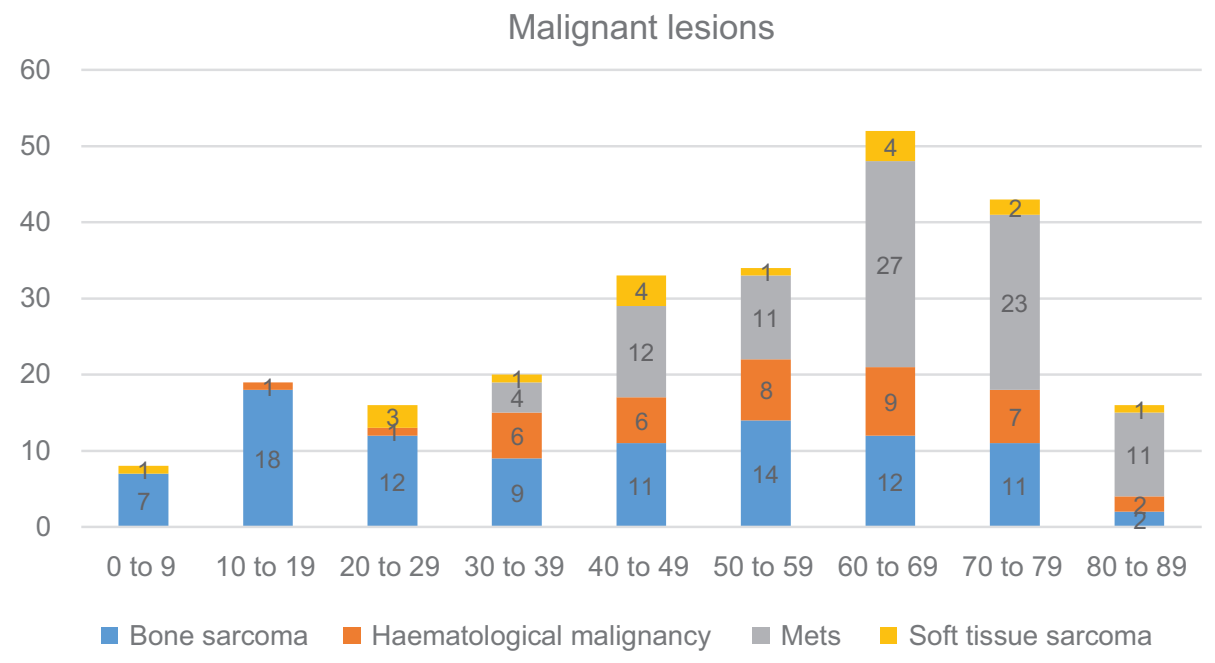

Figure 1. The subtypes of malignant lesions of the scapula.

Due to the relative infrequency of these lesions, we set out to review all the lesions referred to our national sarcoma unit arising from the scapula or periscapular tissue and look at various demographic factors in association with these lesions. We aim to provide some diagnostic guidance when these factors are considered but management in multidisciplinary team setting and biopsy still remain the gold standard. This current study does not include clinical outcomes of these lesions.

\section{Methodology}

A retrospective review of a prospectively held oncology database at our institution was conducted and all patients presenting with a suspicious scapula lesion over a period of 30 years (1985-2015) were included in this study. Data including patient demographics, histological diagnosis, site and laterality was collected and further subgroup analysis was performed for different diagnosis and age groups (in decades).

\section{Results}

A total of 418 patients were found to be eligible for inclusion in the study. All these patients had been referred to us for a "suspicious" lesion in or around the scapula and were discussed in our multidisciplinary team meeting with a review of their clinical and radiological pictures. The median age for the whole cohort was 47 years (range 0-88), and 60\% $(n=249)$ of the study population were male. Fifty-two percent $(n=216)$ of the patients had involvement of the right-sided scapula.

One hundred and thirty-two (32\%) lesions were found to be of soft tissue origin and $286(68 \%)$ were osseous. Overall, $40 \%$ $(n=169)$ of lesions were benign, $57 \%(n=240)$ were malignant and $2 \%(n=9)$ of the lesions were reported as being borderline (Table 1). Females had a slightly higher percentage of malignant lesions than males (62\% vs. $55 \%)$.

Eighty-five percent $(n=96)$ of all sarcomas were osseous in origin and $15 \%(n=17)$ originated from soft tissues. Soft tissue tumours were more likely to be benign $(n=50)$ than malignant $(n=17)$ whereas the number of benign and primary malignant osseous lesions was similar (102 vs. 96, respectively).

\section{Subgroup analysis}

\section{Benign lesions}

Forty-two percent $(n=177)$ of all the scapular lesions were benign of which $58 \%(n=102)$ were osseous and $42 \%$ 
Table 2. Most common benign lesions according to age.

\begin{tabular}{ll}
\hline Age category & \multicolumn{1}{c}{ Most common benign lesion diagnosis } \\
\hline $0-9$ & Osteochondroma $(64 \%, n=16)$ \\
$10-19$ & Osteochondroma $(48 \%, n=15)$ \\
$20-29$ & Osteochondroma $(60 \%, n=18)$ \\
$30-39$ & Osteochondroma $(50 \%, n=12)$ \\
$40-49$ & Non-oncological $(29 \%, n=6)$ \\
$50-59$ & Non-oncological $(18 \%, n=3)$, lipoma $(18 \%, n=3)$ \\
$60-69$ & Non-oncological $(24 \%, n=4)$, \\
& elastofibroma $(24 \%, n=4)$ \\
$70-79$ & Lipoma $(30 \%, n=3)$, elastofibroma $(30 \%, n=3)$ \\
$80-89$ & Osteochondroma $(50 \%, n=1)$, \\
& non-oncological $(50 \%, n=1)$ \\
\hline
\end{tabular}

Table 3. Commonest benign bone tumours of the scapula.

\begin{tabular}{lc}
\hline Subtype of benign bone tumour & Number of cases \\
\hline Osteochondroma & 71 \\
Aneurysmal bone cyst & 7 \\
Eosinophilic granuloma & 6 \\
Fibrous dysplasia & 3 \\
Arteriovenous malformation & 2 \\
Giant cell tumour of bone & 2 \\
Osteoid osteoma & 2 \\
Others & 9 \\
Total & 102 \\
\hline
\end{tabular}

Table 4. Commonest benign soft tissue tumours of the scapula.

\begin{tabular}{lc}
\hline Subtype of benign soft tissue tumour & Number of cases \\
\hline Lipoma & 13 \\
Elastofibroma & 10 \\
Fibromatosis & 7 \\
Enchondroma & 4 \\
Intramuscular lipoma & 4 \\
Ganglion & 2 \\
Others & 10 \\
Total & 50 \\
\hline
\end{tabular}

$(n=75)$ were soft tissue in origin (Figure 1). Osteochondroma was the commonest benign osseous tumour $(70 \%, n=71)$ whereas the commonest soft tissue tumours were lipoma, elastofibroma and fibromatosis (Tables 2-4). Nononcological diagnoses made up $14 \%(n=25)$ of the benign lesions.

\section{Malignant lesions}

Fifty-eight percent $(n=241)$ of all the lesions were malignant of which 113 were primary sarcomas, 88 metastasis and 40 were haematological malignancies (Figure 1 and Table 1).

Chondrosarcoma was the most common primary malignant bone tumour $(45 \%, n=43)$ followed by Ewing's sarcoma $(25 \%, n=24)$ (Table 5). Of the 17 STS, a wide range of histological diagnosis was reported and the commonest
Table 5. Most common bone sarcomas according to age.

\begin{tabular}{ll}
\hline Age category & Most common bone sarcoma diagnosis \\
\hline $0-9$ & Ewing's $(100 \%, n=7)$ \\
$10-19$ & Ewing's $(67 \%, n=12)$ \\
$20-29$ & Chondrosarcoma $(75 \%, n=8)$ \\
$30-39$ & Chondrosarcoma $(78 \%, n=7)$ \\
$40-49$ & Chondrosarcoma $(82 \%, n=9)$ \\
$50-59$ & Chondrosarcoma $(64 \%, n=9)$ \\
$60-69$ & Chondrosarcoma $(42 \%, n=5)$ \\
$70-79$ & Chondrosarcoma $(27 \%, n=3)$, \\
& sarcoma unspecified $(27 \%, n=3)$ \\
$80-89$ & Chondrosarcoma $(50 \%, n=1)$, \\
& secondary osteosarcoma $(50 \%, n=1)$ \\
\hline
\end{tabular}

\section{Soft tissue sarcoma subtypes}

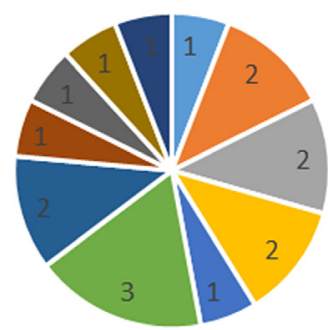

$$
\begin{array}{ll}
\text { n Ewings } & \text { " Fibrosarcoma } \\
\text { " Leiomyosarcoma } & \text { " Liposarcoma } \\
\text { n Malignant epithelioid tumour } & \text { " MFH } \\
\text { - MPNST } & \text { " Myxoid chondrosarcoma } \\
\text { = Rhabdomyosarcoma } & \\
\text { - Undifferentiated pleomorphic sarcoma } &
\end{array}
$$

Figure 2. The subtypes of soft tissue sarcomas of the scapula.

reported tumour was malignant fibrous histiocytoma, now called high grade undifferentiated pleomorphic sarcoma $(18 \%, n=3)$ (Figure 2).

Lungs, kidneys and adenocarcinoma of unknown origin, respectively, were the commonest primary sites for metastasis. Forty-three percent $(n=17)$ of all the haematological malignancies in our series were non-Hodgkin's lymphomas followed by plasmacytomas in $28 \%(n=11)$ of the cases.

\section{Diagnosis according to age}

The commonest age for presentation of any lesion in scapula was in the 6th decade $(n=69)$ (Table 1$)$. In the first decade of life the percentage of benign conditions was $76 \%$ $(n=25)$ but this falls to just $11 \%(n=2)$ by the 9 th decade. Over the first four decades of life $62 \%(n=104)$ of lesions were benign, and were predominantly benign bone tumours $(n=87)$ of which 70\% $(n=61)$ were osteochondromas (Table 2). Eighty-six percent $(n=61)$ of all osteochondromas were found in the first four decades of life. Between the 5th 

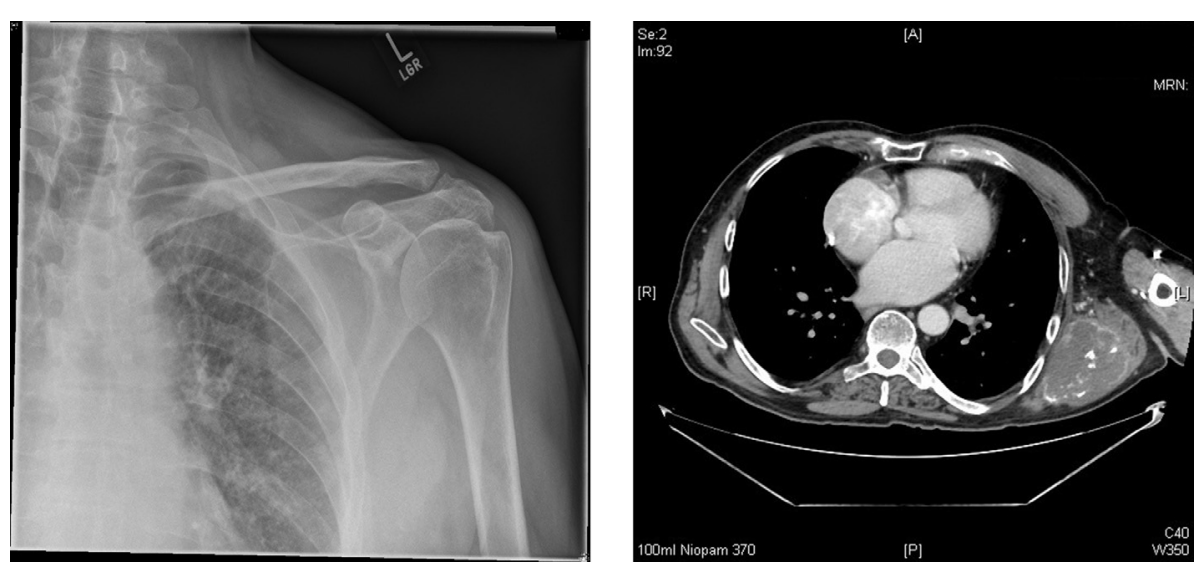

Figure 3. Plain radiograph of left shoulder girdle only showing some soft tissue swelling around the lateral border of the scapula whereas the CT scan demonstrates a destructive lesion consistent with chondrosarcoma.
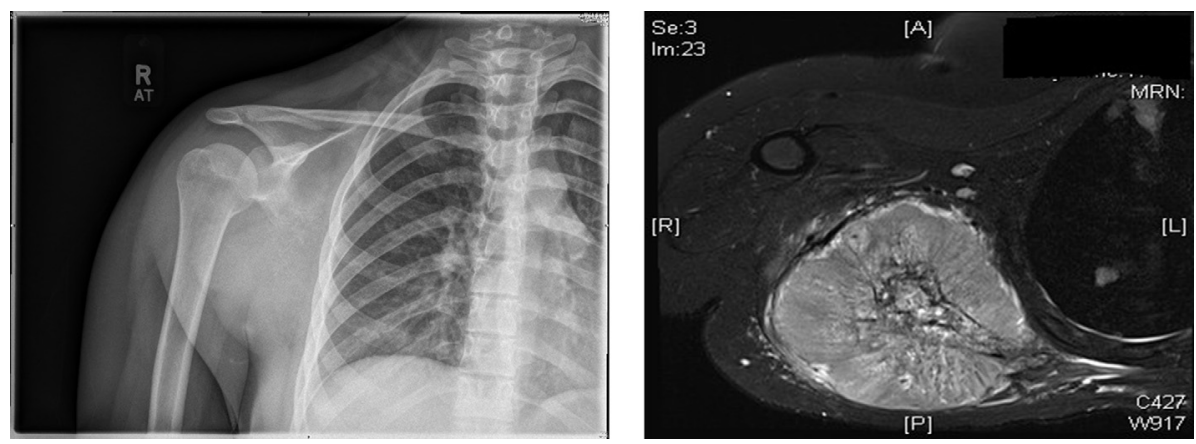

Figure 4. Plain radiograph demonstrating a destructive lesion of the right scapula confirmed with axial view of MRI scan showing a large soft tissue component to the scapular lesions consistent with Ewing's sarcoma.

and 9 th decade just $25 \%(n=60)$ of lesions were found to be benign.

Between the 5th and 8th decade benign soft tissue tumours were the commonest benign lesion $(55 \%, n=36)$. The most common subdiagnosis between the 5th and 9th decade is non-oncological diagnosis (Table 2).

The percentage of malignant conditions steadily increases with increasing age (Figure 1). The number of malignancies increases from the 1st decade $(n=8)$ to a peak in the 7 th decade $(n=49)$. Primary bone sarcomas were distributed almost equally across the age groups (median $=11$, IQ range $=3$ ) however the subdiagnosis of bone sarcoma varies by age. In the first four decades the most predominant type of malignant lesion is bone sarcoma $(73 \%, n=46)$. Chondrosarcoma mainly presented in the 3rd-6th decades and accounted for $61 \%(n=43)$ of bone sarcomas in patients above 20-year age group. Ewing's sarcoma accounted for $25 \%$ $(n=24)$ of bone sarcomas and $79 \%(n=19)$ of these were found in the first two decades of life (Table 5).

Metastases first appeared in patients aged over 30 years and were the most predominant type of lesion in the 7th-9th decades of life $(44 \%, n=61)$. This shows a trend from sarcomas towards metastasis with increasing age. There were a total of 40 haematological malignancies, with $60 \%(n=24)$ in the 6th-8th decades. Soft tissue sarcomas only made up 7\% $(n=17)$ of the malignant lesions and do not appear to affect any single age group predominantly (median $=1$, IQ range $=2$ ) (Figure 1).

\section{Discussion}

The rarity of suspicious lesions of the scapula along with its variable vasculature and difficulty to biopsy increases the challenges associated with the diagnostic workup. There is paucity of published literature on these lesions but a smaller series of 68 lesions found more than one third to be osteochondromas, a quarter to be chondrosarcomas and one third to be a number of other benign and malignant entities [8].

Fifty-seven percent $(n=240)$ of all the lesions in our case series were found to be malignant, consistent with previous research showing that primary bone tumours of the scapula are more likely to be malignant than benign [4]. The percentage of malignant conditions steadily increases with increasing age from $24 \%(n=8)$ in the first decade of life to $89 \%(n=16)$ in the 9 th decade. This demonstrates an increased risk of lesions being malignant with increased age. 


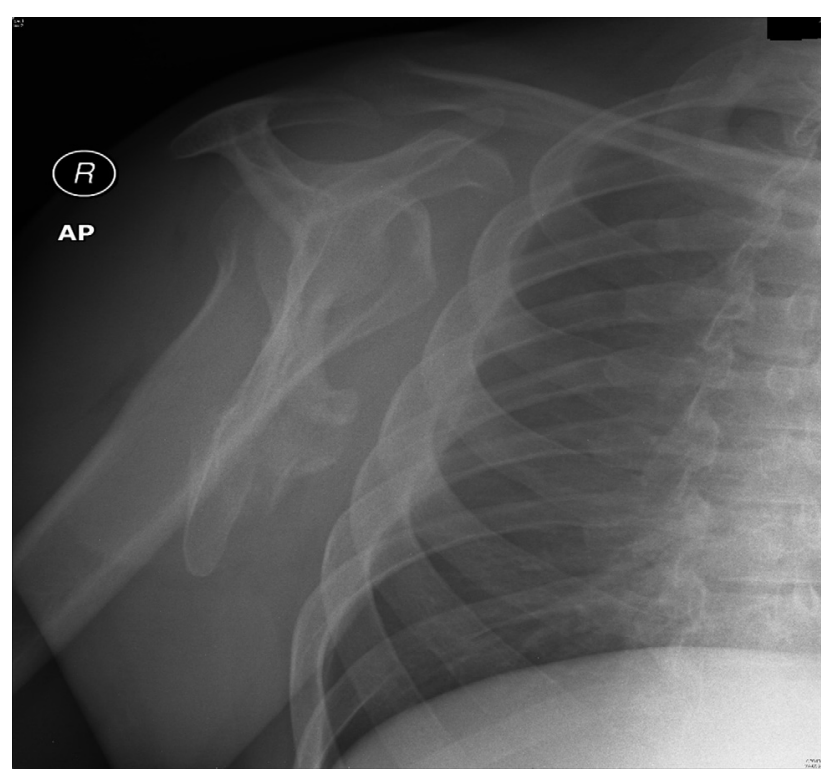

Figure 5. Lateral view of plain radiograph of right scapula demonstrating a broad based pedunculated osteochondroma.

Of the 96 bone sarcomas arising from the scapula, the three most common subtypes were found to be chondrosarcoma $(n=43)$, Ewing's sarcoma $(n=24)$ and osteosarcoma $(n=19)$ which is similar to previously reported studies (Figures 3 and 4). The incidence of osteosarcomas however shows a bimodal distribution with peaks in the 2nd decade of life $(n=4)$ as well as in the 6th-7th decades $(n=9)$. Primary osteosarcomas account for the early age cases but osteosarcomas in the later ages are secondary to Paget's disease and previous radiotherapy [2].

Chondrosarcoma is the second most common primary sarcoma of bone [9]. Only $10 \%(n=43)$ of our cases were found to be chondrosarcomas which is a lower proportion than that found in a study by Brtková et al. [8]. In our series we found that chondrosarcomas were common in the 3rd-6th decades, whereas other studies have found the 4 th- 7 th decades as commonest [8]. This however, is not significantly different as we expect chondrosarcomas to be common in any age after 30 .

Seventy-nine percent $(n=19)$ of Ewing's sarcomas were found in the first two decades of life which is as expected and reported the same by other studies [2].

The distribution of Ewing's sarcoma was almost the same in different genders in our series whereas another study has reported this to be 1.6:1 (male vs. female) [10]. Two cases of Ewing's sarcoma were diagnosed in patients over the age of 30 which is a rare occurrence [2].

We also found that the most common soft tissue sarcomas were high grade undifferentiated pleomorphic sarcoma, previously called malignant fibrous histiocytoma (MFH), liposarcoma and leiomyosarcoma which is similar to what has been reported in the literature before [2]. Osteochondromas made up $17 \%(n=71)$ of all our cases which is lower than the proportion found in a similar study [8]. Osteochondromas were predominantly found in the first four decades of life $(n=61)$ and therefore the difference in proportion may reflect the differences in age between the two cohorts (Figure 5).

Bones are commonly affected by metastatic cancer and metastases are the most common cause of malignant bone lesions [11]. Breast and prostate are the most common malignancies to disseminate to bone and these predominantly affect the axial skeleton, however the scapula can also be affected [11]. Our analysis of scapula metastases found lung, renal and adenocarcinoma of unknown origin to be the most common primary sites.

\section{Conclusions}

Our analysis of this large series has highlighted certain features of the epidemiology of scapula lesions. We found that more than half of all the lesions are malignant and the rate of malignant lesions increases with increasing age. A wide variety of malignant and benign conditions can affect the scapula and this along with the difficult location and peculiar anatomy of this bone can make diagnosis challenging. We recommend a low threshold for imaging and biopsy of suspicious lesion of the scapula and recommend that they should be dealt with in a specialist sarcoma unit in order to receive the correct systemic diagnostic work up in a multidisciplinary team setting.

\section{Limitations}

Retrospective study over a 30 -year period.

\section{Conflict of interest}

The author(s) declare no conflict of interest in relation with this paper.

\section{References}

1. Puchner SE, Panotopoulos J, Puchner R, Schuh R, Windhager R, Funovics PT (2014) Primary malignant tumours of the scapula-a review of 29 cases. Int Orthop 38(10), 2155-2162.

2. Malawer MM, Sugarbaker PH (2007) Musculoskeletal cancer surgery, treatment of sarcomas and allied diseases, Dordrecht, Netherlands, Springer Science \& Business Media.

3. Dahlin DC (1957) Bone tumors. Springfield, Charles C Thomas, pp. 114-128

4. Shahid M, Varshney M, Maheshwari V, et al (2011) Ewing's sarcoma of scapula: a rare entity. BMJ Case Rep 2011.

5. Charles AR, Frederick AM, Michael AW, Steven BL (2009) The shoulder. Philadelphia, Saunders, Elsevier.

6. Mavrogenis AF, Mastorakos DP, Triantafyllopoulos G, et al. (2009) Total scapulectomy and constrained reverse total shoulder reconstruction for a Ewing's sarcoma. J Surg Oncol 100, 611-615.

7. Mayil Vahanan N, Mohanlal P, Bose JC, et al. (2007) The functional and oncological results after scapulectomy for scapular tumours: 2-16-year results. Int Orthop 31, 831-836. 
8. Brtková J, Nidecker A, Zídková H, Jundt G. (1999) Tumours and tumour-like lesions of scapula. Acta Medica (Hradec Kralove) 42(3), 103-110.

9. Pant R, Yasko AW, Lewis VO, Raymond K, Lin PP. (2005) Chondrosarcoma of the scapula: long-term oncologic outcome. Cancer 104(1), 149-158.
10. Asif N, Khan AQ, Siddiqui YS, Mustafa H. (2010) Metastasis from scapular Ewing's sarcoma presenting as sutural diastasis: An unusual presentation. Int J Shoulder Surg 4(1), 18-21.

11. Jasmin C, Capanna R. (2005) Textbook of bone metastases. Chichester, West Sussex, England, John Wiley \& Sons.

Cite this article as: Khan Z, Gerrish AM \& Grimer RJ (2016) An epidemiological survey of tumour or tumour like conditions in the scapula and periscapular region. SICOT J, 2, 34 